A N N A L E S Annales de Bretagne et des Pays de l'Ouest

\title{
Le port de Nantes au XVIIIe siècle. Construction d'une aire portuaire
}

\section{Gilbert Buti}

\section{(2) OpenEdition}

\section{Journals}

\section{Édition électronique}

URL : http://journals.openedition.org/abpo/2700

DOI : $10.4000 /$ abpo. 2700

ISBN : 978-2-7535-3304-2

ISSN : 2108-6443

Éditeur

Presses universitaires de Rennes

Édition imprimée

Date de publication : 30 décembre 2013

Pagination : 209-212

ISBN : 978-2-7535-3302-8

ISSN : 0399-0826

\section{Référence électronique}

Gilbert Buti, « Le port de Nantes au XVIIIe siècle. Construction d'une aire portuaire », Annales de Bretagne et des Pays de l'Ouest [En ligne], 120-4 | 2013, mis en ligne le 30 décembre 2013, consulté le 30 avril 2019. URL : http://journals.openedition.org/abpo/2700

Ce document a été généré automatiquement le 30 avril 2019.

(c) Presses universitaires de Rennes 


\title{
Le port de Nantes au XVIIIe siècle. Construction d'une aire portuaire
}

\author{
Gilbert Buti
}

\section{RÉFÉRENCE}

MICHON, Bernard, Le port de Nantes au XVIII siècle. Construction d'une aire portuaire, Rennes, PUR, coll. « Histoire », 2011, 397 p.

1 L'ouvrage de Bernard Michon reprend en partie, car centré sur le seul XVIII ${ }^{\mathrm{e}}$ siècle, sa thèse de doctorat d'histoire présentée devant l'université de Nantes en 2005. Le texte, fort de 339 pages jalonnées de tableaux, cartes, graphiques et gravures, est complété par des annexes qui fournissent des éléments sur les trafics portuaires nantais au XVIII ${ }^{\mathrm{e}}$ siècle, période de la "prospérité triomphante " (Jean Meyer) du port de Nantes qui se range alors parmi les quatre grands complexes portuaires du royaume à la fin de l'Ancien Régime, à un moment où la France joue un rôle majeur dans le système maritime et colonial mondial. Dans cette recherche, placée dans le sillage des travaux de Jean Tanguy et de Jean Meyer, et plus récemment de Murielle Bouyer, l'auteur s'attache à analyser l'extension de l'espace portuaire nantais, à définir le domaine littoral sur lequel le grand port exerce son influence, voire sa domination, et à décrypter l'organisation interne de ce territoire en pointant les modifications intervenues au cours d'une période qui place le port parmi les plus importants de la façade atlantique. S'interrogeant sur l'impact que la forte croissance du pôle nantais a pu exercer sur la configuration d'un vaste ensemble portuaire, Bernard Michon analyse également la structure et les complémentarités fonctionnelles de cette construction en accordant une place aux rapports économiques et techniques qui lient le grand port de la Loire aux autres ports de l'estuaire et en portant attention aux acteurs ainsi qu'à la circulation des capitaux qui permettent la mobilisation des flottes de diverse nature.

2 Définir la zone d'influence d'un grand port conduit l'auteur à préciser d'entrée de jeu, et sans polémique inutile, le choix retenu de la dénomination d'aire portuaire qui renvoie à 
une logique géographique d'organisation de l'espace; il retient également la définition de complexe portuaire en convoquant pour cela celles proposées par Claude Nières et André Lespagnol, à savoir un ensemble de sites organisés autour d'un pôle principal et pouvant être regardés de l'extérieur comme constituant un seul et même ensemble portuaire participant, en l'occurrence, à deux types d'aires : estuarien et linéaire.

Bernard Michon apporte, par son étude, une meilleure connaissance de l'articulation des trafics entre les ports de l'aire nantaise et définit les différents flux qui existent entre le port majeur ou " port source » et les ports secondaires ou « obliques » longtemps négligés par l'historiographie. Il ne s'agit donc ni d'une étude sur les horizons commerciaux nantais, ni sur la formation d'une élite négociante, bien que ces éléments ne soient pas absents, mais d'une analyse de l'ensemble portuaire sollicité par le négoce nantais, une organisation certes souvent mentionnée mais de manière très générale.

4 La réflexion est articulée autour de quatre axes qui forment les parties d'importance inégale de l'ouvrage. La première présente «l'aire portuaire de Nantes face au défi colonial», la deuxième s'arrête sur «la grande pêche morutière comme armement délégué aux ports secondaires ", la troisième analyse, à travers le mouvement du port de Nantes, la «zone d'échanges économiques privilégiés et l'importance de Nantes dans l'activité des ports environnants » et la dernière partie, plus brève, est consacrée aux «flottilles de cabotage au service du pôle de commandement nantais ».

Bernard Michon fait une présentation, à la fois claire et dense, des différents éléments qui composent le territoire d'influence portuaire de Nantes, en distinguant trois espaces concentriques emboîtés selon trois échelles d'observation. En premier lieu, figure l'organisme portuaire estuarien de Nantes, composé de plusieurs ports, inféodés ou non au grand port de la Loire, avec une distribution des trafics entre cabotage à court rayon, grand cabotage européen et trafic colonial. L'auteur opère également une distinction entre les différentes composantes de ce premier cercle. Le port de Nantes, "port de commandement ", de la Poterne à l'Hermitage, en débordant vers la Fosse et la Chézine, est avant tout un port de gabarres d'estuaire qui permettent à la cité ligérienne de contrôler une partie non négligeable du trafic maritime de cabotage à l'échelle du royaume, voire de l'Europe, et surtout de conserver une fonction d'entrepôt. Par ailleurs, le long des deux rives de la Loire maritime, Nantes dispose de plusieurs avant-ports spécialisés: le duo formé par Couëron-Port Launay, en expansion au XVIII ${ }^{\mathrm{e}}$ siècle, constitue le port des gros porteurs du cabotage intra-européen tandis que Le Pellerin, port de mouillage et de réparations navales, apparaît en déclin; Paimbœuf, qui bénéficie de bonnes conditions nautiques mais qui s'appuie de plus en plus sur son avant-port Mindin, est le véritable port pour le trafic au long cours, notamment pour le commerce antillais; Saint-Nazaire, port de relâche à l'entrée de l'estuaire, abrite la station des pilotes et lamaneurs et connaît peu d'évolution au cours de la période étudiée. Nantes, qui refuse longtemps de nommer un capitaine de port à Paimbœuf, conserve le pouvoir commercial, les institutions du consulat et de l'amirauté, et contraint les capitaines à venir y accomplir leurs démarches.

6 Au-delà de l'estuaire, l'aire d'approvisionnement en produits vivriers (céréales, sel, poissons) compose un deuxième cercle, de Quimper et Penerf, au nord, à l'estuaire extérieur du Croisic et aux Sables-d'Olonne au sud. Ces ports, qui possèdent leur propre flotte, sont parfois spécialisés dans des fonctions de service, ainsi ceux des îles d'Yeu et du golfe de Rhuys, dans la mesure où les Nantais, comme on a pu l'observer dans d'autres places portuaires, ne serait-ce qu'à Marseille, ont fait le choix de ne pas se livrer à ces 
échanges de proximité. Au reste, ces ports du deuxième cercle ne sont pas exclusivement tournés vers Nantes; les ports de la baie de Bourgneuf, Vannes et Les Sables-d'Olonne ne sont pas totalement sous la tutelle du marché nantais dans la mesure où les armements américains qu'ils sont autorisés à pratiquer limitent cette influence. En élargissant leur marge de manœuvre et en jouant sur les différentes aires portuaires, ils exportent également du sel vers l'Europe du nord-ouest ou des grains vers Bordeaux et La Rochelle. Le chevauchement de ces différentes aires conduit nécessairement à une compétition entre les ports de Nantes, La Rochelle et Bordeaux, soucieux de contrôler au mieux leurs territoires, qui mériterait d'être analysée en profondeur. La question des limites de l'aire portuaire nantaise se pose également lorsqu'elle se heurte à l'avant-pays maritime de Lorient, qui dispose certes des ressources de son hinterland, mais qui dépend également de celles de l'arrière-pays nantais et de la clientèle nantaise pour l'écoulement d'une partie des produits asiatiques. Bernard Michon hésite cependant à faire de Lorient, port de la compagnie des Indes, une des composantes de l'aire portuaire de la cité ligérienne ; Lorient exerce une attraction sur les ports du littoral sud de la Bretagne; toutefois, comme le reconnaît Gérard Le Bouëdec, «Lorient sans Nantes n'était pas viable ». Dans cette « zone d'échanges économiques privilégiés » à l'étude accompagnée de cartes claires et bienvenues, il aurait sans doute été bon de justifier le choix des années de référence (1702-1732-1772).

7 À une autre échelle enfin, Bernard Michon montre comment les activités transatlantiques conduisent à une reconfiguration de l'aire portuaire nantaise. Dans le système morutier, analysé dans la deuxième partie de l'ouvrage, on observait un partage des tâches : Nantes étant le marché de gros, Le Croisic et plus encore Les Sables-d'olonne des ports d'armement relativement autonomes. Dans la première moitié du XvIII ${ }^{e}$ siècle, avec le développement des trafics antillais et le déclin du microcapitalisme morutier, Nantes devient le centre unique de l'impulsion économique. Le rachat de navires morutiers des ports de pêche majeurs (Le Croisic, Pornic, Les Sables-d'Olonne), lors des années de conflit ou de reprise de l'activité maritime d'après-guerre, et leur affectation sur la route des Antilles, ainsi que l'orientation des capitaux nantais vers l'armement antillais conduisent à faire du trafic antillais une affaire essentiellement nantaise, avec un haut négoce qui a peu puisé dans le réservoir des élites locales des ports de l'aire. Bernard Michon considère cependant que si le déclin, voire l'arrêt des armements morutiers par les ports situés à proximité de Nantes, fait "vaciller le système », les données recueillies pour le XvIII ${ }^{\mathrm{e}}$ siècle témoignent du maintien d'une activité morutière dans les ports de la baie de Bourgneuf et surtout aux Sables-d'olonne qui reste majoritairement aux mains d'armateurs locaux. Pour l'auteur, les causes profondes du déclin de l'activité morutière sont à trouver dans la situation interne des ports environnants qui connaissent la réduction de leur élite marchande et son non renouvellement ; c'est dans ces directions qu'il faut chercher, selon lui, «les motifs décisifs du déclin des Sables-d'olonne » plutôt que dans le désengagement des créanciers nantais des entreprises d'armement ou dans un retrait volontaire des Sablais de la pêche morutière. Dans cette nouvelle configuration, la dynamique nantaise retient les capitaux et transforme son aire portuaire en bassin de recrutement de main-d'œuvre pour les équipages comme l'a observé Murielle Bouyer (Murielle Bouyer, Les marins de la Loire dans le commerce maritime nantais au XVIII siècle, Rennes, PUR, 2008). Peut-être était-il nécessaire, même si l'objectif de l'auteur était autre, de dépasser le jeu des rapports entre Nantes et ses proches voisins pour rappeler le rôle essentiel de partenaires et acteurs économiques plus lointains, comme ces gens de 
finances de Paris ou d'Orléans, qui interviennent dans le négoce nantais et qui ne sont sans doute pas totalement étrangers à l'essor de la place de la Loire dans la première moitié du siècle étudié ?

8 Se trouve également posée, en articulant les trafics lointains aux échanges intraeuropéens, la délicate question du rapport entre le commerce colonial et le cabotage. Ainsi, à titre d'exemple, une part des échanges effectués avec les Provinces-Unies permet aux Nantais de se pourvoir en produits nécessaires à la traite atlantique (quincaillerie, textiles, cauris). Si le commerce avec les Îles d'Amérique est le moteur, par la concentration et la redistribution des marchandises, de la croissance d'un trafic de grand cabotage européen, force est de reconnaître que le cabotage possède sa propre dynamique et que le développement urbain anime une circulation caboteuse autonome. La géographie des trafics à court rayon est bien traitée, néanmoins la $4^{\mathrm{e}}$ partie dédiée aux flottilles de cabotage parait un peu plaquée sur la construction générale ; elle pouvait être distribuée autrement au fil de la démonstration sans prendre cette forme un peu statique, très descriptive, ou être davantage développée dans une partie autonome, plus dynamique et insérée différemment dans l'architecture de la publication.

Il n'en reste pas moins que l'ouvrage de Bernard Michon décrit l'anatomie d'un système portuaire en cours de recomposition au gré de la conjoncture et suit l'impact que la croissance du pôle nantais a eu sur l'organisation du territoire. Le modèle nantais d'aire portuaire associant un pôle de commandement, un estuaire et le proche littoral ne prétend cependant pas résumer à lui seul toutes les formes d'organisation d'espace d'influence portuaire; si le complexe Rouen-Le Havre, voire celui de Bayonne avec les ports du Labourd, sont proches de celui analysé par Bernard Michon, le cas de Bordeaux s'en éloigne dans la mesure où l'ampleur de la Gironde permet à celui-ci de se passer d'un grand avant-port comme Paimboeuf. Au reste, dans d'autres configurations spatiales et en restant sur les rivages du royaume qu'il conviendrait toutefois de dépasser, SaintMalo, Dunkerque et Marseille offrent des organisations différentes. Bernard Michon apporte assurément avec cet ouvrage une contribution à la meilleure connaissance de la dynamique de la façade atlantique au XVIII ${ }^{\mathrm{e}}$ siècle, et plus largement un élément à la compréhension du fonctionnement des façades maritimes. 